\title{
Karhunen-Loève Expansion of the WSSUS Channel Output and Its Application to Efficient Simulation
}

\author{
Kun-Wah Yip, Member, IEEE, and Tung-Sang Ng
}

\begin{abstract}
This paper derives a Karhunen-Loève (K-L) expansion of the time-varying output of a multipath Rayleigh fading wide-sense-stationary uncorrelated-scattering (WSSUS) channel. It is shown that under the same mean-squared error condition, the number of terms required by the truncated $\mathrm{K}-\mathrm{L}$ expansion is less than that of the series expansion obtained by using the discrete-path approximation of the channel so that simulation using the $\mathrm{K}-\mathrm{L}$ expansion is more efficient. This computational advantage becomes more significant as higher simulation accuracy is required. The derived $\mathrm{K}-\mathrm{L}$ expansion is applied to develop an efficient simulation technique for digital transmission over a multipath Rayleigh fading WSSUS channel using an optimum receiver. We show that the proposed technique requires shorter computation time than two other known simulation techniques.
\end{abstract}

Index Terms-Efficient simulation technique, Karhunen-Loève expansion, multipath Rayleigh fading WSSUS channel.

\section{INTRODUCTION}

$\mathbf{I}$ $\mathrm{N}$ this paper, a new methodology for efficient simulation of digital communications over a multipath Rayleigh fading wide-sense-stationary uncorrelated-scattering (WSSUS) channel is proposed. This methodology is based on the Karhunen-Loève $(\mathrm{K}-\mathrm{L})$ expansion of the time-varying channel output. The $\mathrm{K}-\mathrm{L}$ expansion of a random process is well known for its optimal truncation property [1], [2, ch. 4.7]. That is, the $\mathrm{K}-\mathrm{L}$ expansion requires the minimum number of terms among all possible series expansions in representing a random process for a given mean-squared error. In generating sample functions of a Gaussian random process in simulation, a smaller number of terms in the series expansion results in a smaller computation load. Thus, the optimal truncation property of the $\mathrm{K}-\mathrm{L}$ expansion results in the minimization of computation load required to generate a Gaussian random process in simulation. This computational advantage motivates the development of the present work.

We will show later that the channel output due to a symbol pulse is given by the convolution between the time-varying channel impulse response, $h(\tau ; t)$, and the symbol waveform, $\psi(\tau)$. That is, the resultant channel output $\Omega(\tau ; t)$ is given by $\Omega(\tau ; t)=\int h\left(\tau^{\prime} ; t\right) \psi\left(\tau-\tau^{\prime}\right) d \tau^{\prime}$, which is a two-dimensional random process in both time $t$ and delay $\tau$. In this paper, we derive the $\mathrm{K}-\mathrm{L}$ expansion of $\Omega(\tau ; t)$ in the dimension $\tau$. We consider only the case that the WSSUS channel is separable

Manuscript received April 10, 1996; revised October 9, 1996. This work was supported by the Hong Kong Research Grants Council and by the University Research Committee of The University of Hong Kong.

The authors are with the Department of Electrical and Electronic Engineering, The University of Hong Kong, Pokfulam Road, Hong Kong.

Publisher Item Identifier S 0733-8716(97)02669-3. and Gaussian. A separable WSSUS channel is one whose fading and channel dispersion characteristics are independent. Based on the assumption of a separable Gaussian WSSUS channel it will be shown that coefficients of the resultant $\mathrm{K}-\mathrm{L}$ expansion are uncorrelated complex Gaussian random processes in $t$, and that statistical properties of these random processes are determined by the Doppler power spectrum of the channel. Then $\Omega(\tau ; t)$ can be efficiently computed by applying a known method to generate sample functions of these uncorrelated random processes, followed by substituting the generated sample functions as coefficients into the $\mathrm{K}-\mathrm{L}$ expansion.

Previous literature closely related to the present work was given by Clark et al. [3], and Clark [4]. In [3] and [4], the K-L expansion was derived for the Fourier transform of $\Omega(\tau ; t)$ on the $\tau$ variable. ${ }^{1}$ It follows that the resultant $\mathrm{K}-\mathrm{L}$ expansion is expressed in the frequency domain, while the one derived in this paper is in the $\tau$ domain. The frequency-domain $\mathrm{K}-\mathrm{L}$ expansion was used for analytical derivation of the matched filter bound in [3] and for the implementation of simulation in a subsequent work [5] (see footnote 3 of [5]). Although not explicitly stated in [5], the frequency-domain $\mathrm{K}-\mathrm{L}$ expansion possesses the optimal truncation property as does the $\tau$-domain $\mathrm{K}-\mathrm{L}$ expansion that is derived in this paper so that simulation using the frequency-domain $\mathrm{K}-\mathrm{L}$ expansion is also efficient. The choice of which $\mathrm{K}-\mathrm{L}$ expansion to use for simulation depends on its suitability to one's application. For instance, one may prefer using the frequency-domain ( $\tau$-domain) $\mathrm{K}-\mathrm{L}$ expansion if system transfer functions, such as the channel impulse response, symbol pulse shape, etc., are represented in the frequency domain ( $\tau$ domain).

Other related published literature was given by Fechtel [6], Crespo and Jiménez [7], and Visintin [8]. In [6], the channel output $\Omega(\tau ; t)$ was approximated in the dimension $\tau$ by a series expansion with a specific choice of basis functions. It was shown that under typical conditions the number of basis functions in the series expansion was less than the number of discrete multipaths in approximating the channel impulse response (a commonly used technique in simulation) so that a reduction in the computation load could be achieved. However, in many cases the series expansion of [6] does not mean-square converge to the channel output even with an infinite number of basis functions, and this results in a loss

\footnotetext{
${ }^{1}$ The authors of [3] and [4] actually considered $\Omega(\tau)$ in the derivation so that coefficients of the expansion are uncorrelated Gaussian random variables rather than random processes in $t$. In spite of slight confusion that may arise, we write $\Omega(\tau ; t)$ instead of $\Omega(\tau)$ for the sake of notational consistency with the present work.
} 
of modeling accuracy. On the other hand, the mean-squared convergence property of the $\mathrm{K}-\mathrm{L}$ expansion is well known and conditions of convergence are usually satisfied in practical situations. In addition, because of the optimal truncation property, the $\mathrm{K}-\mathrm{L}$ expansion requires fewer basis functions in representing $\Omega(\tau ; t)$ than the series expansion of [6] under the same mean-squared error condition. Therefore, simulation using the $\mathrm{K}-\mathrm{L}$ expansion requires less computational effort. In [7], it was shown that a complex Gaussian random process could be efficiently generated by its Fourier series expansion. The channel output $\Omega(\tau ; t)$ was then computed by using the discrete-path approximation of the channel, followed by an application of the Fourier series expansion technique to generate the time-varying complex path gain. That is, the discrete-path approximation was used to model $\Omega(\tau ; t)$ in the dimension $\tau$ and the Fourier series expansion technique was proposed for efficient generation of $\Omega(\tau ; t)$ in the dimension $t$. In the present work, we are concerned with modeling $\Omega(\tau ; t)$ in the dimension $\tau$ by the $\mathrm{K}-\mathrm{L}$ expansion. This work is different from the recent work of [8]. In [8], the K-L expansion was derived for a particular complex Gaussian random process characterized by the Jakes Doppler power spectrum. The K-L expansion of [8] is useful for efficient generation of $\Omega(\tau ; t)$ in the dimension $t$.

The derived $\mathrm{K}-\mathrm{L}$ expansion is then used to develop an efficient simulation technique for digital communications via a multipath Rayleigh fading WSSUS channel using an optimum receiver for signal detection. This optimum receiver is a matched filter matched to the combined characteristic of the channel and the symbol pulse shape [9, ch. 6.3]. By modeling the communication system as an equivalent discrete-time model, which can be realized by a finite-impulse-response (FIR) filter with time-variant tap gains, one can efficiently simulate the communication system [10], [11]. We derive the expression of the time-variant tap gain based on the $\mathrm{K}-\mathrm{L}$ expansion of the WSSUS channel output, and show that the proposed technique requires shorter computation time than the one based on the discrete-path approximation of the channel and an earlier simulation technique proposed by Hoeher [10]. The present work is different from the authors' previous work in [11]. In [11], we considered a suboptimum receiver (a matched filter matched to the symbol pulse shape) and the time-variant tap gains were generated based on a linear transformation of uncorrelated complex Gaussian random processes.

The organization of this paper is as follows. Section II describes the WSSUS channel model. The $\mathrm{K}-\mathrm{L}$ expansion of the WSSUS channel output is derived in Section III, and its simulation efficiency is demonstrated in Section IV. Section V develops an efficient simulation technique for digital communications using an optimum receiver, and comparison with other techniques is also given. Finally, conclusions are given in Section VI.

\section{WSSUS CHANNEL MODEL}

Let $h(\tau ; t)$ be the low-pass-equivalent time-varying channel impulse response, where $h(\tau ; t)$ is the channel output at time $t$ when a unit impulse is applied at time $t-\tau$. A multipath Rayleigh fading WSSUS channel can be characterized by the autocorrelation function, given by [12], [9, ch. 7.1]

$$
\frac{1}{2} E\left\{h(\tau ; t) h^{*}(\xi ; t+\Delta t)\right\}=Q_{h}(\tau ; \Delta t) \delta(\xi-\tau)
$$

where $Q_{h}(\tau ; \Delta t)$ is the delay cross-power density function. In the special case of $\Delta t=0, Q(\tau) \triangleq Q_{h}(\tau ; 0)$ is known as the delay power spectrum.

In this paper, it is assumed that the channel dispersion characteristic and the fading characteristic are statistically independent. This assumption is usually satisfied in practical situations because the channel dispersion characteristic most often depends on the scatterer distribution whereas the fading characteristic usually depends on the relative motion between the transmitter and the receiver. Then $Q_{h}(\tau ; \Delta t)$ can be factored into

$$
Q_{h}(t ; \Delta t)=Q(\tau) D(\Delta t)
$$

where $D(\Delta t)$ describes the fading characteristic of the channel and can be computed from the Doppler power spectrum, $S(f)$, by [11]

$$
D(\Delta t)=\frac{\int_{-\infty}^{\infty} S(f) e^{j 2 \pi f(\Delta t)} d f}{\int_{-\infty}^{\infty} S(f) d f} .
$$

\section{KARHUNEN-LOĖVE EXPANSION OF THE ChanNEL OUTPUT}

Consider a digital communication system transmitting a signal over a multipath Rayleigh fading WSSUS channel with the low-pass-equivalent channel impulse response $h(\tau ; t)$. Let $\psi(\tau)$ be the transmitted symbol pulse of length $T_{\psi}$. It is assumed that $\psi(\tau)$ is real and is time-limited for $\tau \in\left[0, T_{\psi}\right)$. Without loss of generality it is also assumed that $h(\tau ; t)=$ $0, \tau \notin\left[0, T_{M}\right)$, where $T_{M}$ is the multipath spread of the channel. If the channel is slowly varying relative to (at least) $T_{M}+T_{\psi}$, convolution of $h(\tau ; t)$ and $\psi(\tau)$ gives the timevarying received symbol pulse shape $\Omega(\tau ; t)$, i.e.,

$$
\Omega(\tau ; t)=\int_{0^{-}}^{T_{M}} h\left(\tau^{\prime} ; t\right) \psi\left(\tau-\tau^{\prime}\right) d \tau^{\prime}, \quad 0 \leq \tau<T_{\Omega}
$$

where $T_{\Omega}=T_{M}+T_{\psi}$ is the length of $\Omega(\tau ; t)$.

It is apparent that $\Omega(\tau ; t)$ is a random process in $t$. In addition, at times $t_{1}, t_{2}, \cdots, t_{m}$, we can regard $\Omega\left(\tau ; t_{1}\right)$, $\Omega\left(\tau ; t_{2}\right), \cdots, \Omega\left(\tau ; t_{m}\right)$ as sample functions of $\Omega(\tau ; t)$ in the parameter $\tau$, so that $\Omega(\tau ; t)$ is also a random process in $\tau$. The autocorrelation function of $\Omega(\tau ; t)$ is given by

$$
\begin{aligned}
& E\left\{\Omega(\tau ; t) \Omega^{*}(\xi ; t+\Delta t)\right\} \\
& \quad=D(\Delta t) \int_{0^{-}}^{T_{M}} 2 Q\left(\tau^{\prime}\right) \psi\left(\tau-\tau^{\prime}\right) \psi\left(\xi-\tau^{\prime}\right) d \tau^{\prime} .
\end{aligned}
$$

The last expression is obtained by substituting (4) into the left hand side of (5) followed by applying (1) and (2). Let

$$
R(\tau, \xi)=\left.E\left\{\Omega(\tau ; t) \Omega^{*}(\xi ; t+\Delta t)\right\}\right|_{\Delta t=0} .
$$


Since $D(0)=1$, the expression of $R(\tau, \xi)$ can be easily obtained from (5). Expanding $\Omega(\tau ; t)$ by the $\mathrm{K}-\mathrm{L}$ expansion in the parameter $\tau$ gives $[2$, ch. 4.6$],[13$, ch. 6.4$]$

$$
\Omega(\tau ; t)=\lim _{N \rightarrow \infty} \Omega_{N}(\tau ; t), \quad 0 \leq \tau<T_{\Omega}
$$

where

$$
\Omega_{N}(\tau ; t)=\sum_{n=1}^{N} w_{n}(t) \phi_{n}(\tau)
$$

and the equality of (7) holds in the sense of mean-squared convergence. The $\phi_{n}(\tau), n=1,2, \cdots, N$, satisfying $\phi_{n}(\tau)=0$ for $\tau \in\left[0, T_{\Omega}\right)$, form a set of orthonormal basis functions. Each of these basis functions is an eigenfunction of the integral equation

$$
\int_{0^{-}}^{T_{\Omega}} R(\tau, \xi) \phi_{n}(\xi) d \xi=\lambda_{n} \phi_{n}(\tau)
$$

where $\lambda_{n}$ is the corresponding eigenvalue. The $w_{n}(t), n=$ $1,2, \cdots, N$, are independent zero-mean complex Gaussian random processes with

$$
E\left\{w_{n}(t) w_{n}^{*}(t)\right\}=\lambda_{n}
$$

Analytical solutions of (9) are known for some forms of $R(\tau, \xi)$ [13], [14]. Numerical solutions can be obtained for a general continuous ${ }^{2} R(\tau, \xi)$ based on Simpson's rule as outlined in [3] and in Appendix I. Interested readers may also refer to [15] for other numerical techniques that solve (9). Since $R(\tau, \xi)$ is real symmetric and nonnegative definite, it follows that $\lambda_{n}$ 's are nonnegative, a result that is expected as indicated by (10). Notice that a knowledge of $E\left\{w_{n}(t) w_{n}^{*}(t+\Delta t)\right\}$ is required to statistically characterize the random process $w_{n}(t)$. From (7) and by the orthonormal property of $\phi_{n}(\tau)$ 's, one gets

$$
w_{n}(t)=\int_{0^{-}}^{T_{\Omega}} \Omega(\tau ; t) \phi_{n}^{*}(\tau) d \tau
$$

Evaluating $E\left\{w_{n}(t) w_{n}^{*}(t+\Delta t)\right\}$ using (11) and noting that $E\left\{w_{n}(t) w_{n}^{*}(t)\right\}$ is given by (10) yields

$$
E\left\{w_{n}(t) w_{n}^{*}(t+\Delta t)\right\}=\lambda_{n} \cdot D(\Delta t) .
$$

As an additional observation, taking Fourier transform on (12) and applying (3) reveals that the power spectral density of $w_{n}(t)$ is proportional to the Doppler power spectrum of the channel.

Although $\Omega(\tau ; t)$ is expressed as an infinite series in (7), it can only be computed with a finite number of terms in simulation. The mean-squared truncation error, given by

$$
E\left\{\varepsilon_{N}^{2}\right\}=E\left\{\int_{0^{-}}^{T_{\Omega}}\left|\Omega(\tau ; t)-\Omega_{N}(\tau ; t)\right|^{2} d \tau\right\}
$$

is useful in controlling the accuracy in simulation. It is known that [2, ch. 4.7]

$$
E\left\{\varepsilon_{N}^{2}\right\}=\int_{0^{-}}^{T_{\Omega}} R(\tau, \tau) d \tau-\sum_{n=1}^{N} \lambda_{n} .
$$

\footnotetext{
${ }^{2}$ The $\mathrm{K}-\mathrm{L}$ expansion is usually applied to simulation where $Q(\tau)$ is continuous or piecewise continuous. In this case $R(\tau, \xi)$ is continuous provided $\psi(\tau)$ is continuous over $\tau \in\left(0, T_{\psi}\right)$.
}

It is important to point out that a truncated $\mathrm{K}-\mathrm{L}$ expansion $\Omega_{N}(\tau ; t)$ can be formed by selecting any $N$ basis functions among all basis functions that satisfy (9). The (optimal) one that yields the smallest $E\left\{\varepsilon_{N}^{2}\right\}$ is the one expanded with the basis functions associated with the first $N$ greatest eigenvalues as indicated by (14). This optimal truncated $\mathrm{K}-\mathrm{L}$ expansion is selected in the efficient computation of $\Omega(\tau ; t)$.

\section{COMPARISON RESULTS}

We first compare the number of terms required in the computation of $\Omega(\tau ; t)$ using the truncated $\mathrm{K}-\mathrm{L}$ expansion and that using the series expansion obtained via the discrete-path approximation of $h(\tau ; t)$. For the discrete-path approximation, $\Omega(\tau ; t)$ is computed by

$$
\Omega(\tau ; t) \approx \sum_{n=1}^{N_{p}} \alpha_{n}(t) \psi\left(\tau-\tau_{n}\right)
$$

where $\alpha_{n}(t)$ and $\tau_{n}$ are the complex path gain and the path delay, respectively, of the $n$th path of the approximate discrete-path channel impulse response, and $N_{p}$ is the number of paths. The complex path gain is computed by $\alpha_{n}(t)=$ $\int_{\tilde{\tau}_{n-1}}^{\tilde{\tau}_{n}} h(\tau ; t) d \tau$ where $\tilde{\tau}_{0}, \tilde{\tau}_{1}, \cdots, \tilde{\tau}_{N_{p}}$ are strictly increasing with $\widetilde{\tau}_{0}=0^{-}$and $\tilde{\tau}_{N_{p}}=T_{M}$, satisfying $\tilde{\tau}_{n-1} \leq \tau_{n} \leq \tilde{\tau}_{n}$. That is, the approximate discrete-path channel impulse response approximates the contribution of $h(\tau ; t)$ for $\tau \in\left[\tilde{\tau}_{n-1}, \tilde{\tau}_{n}\right)$ by a discrete path of complex path gain $\alpha_{n}(t)$ at a delay $\tau_{n}$, where $\tilde{\tau}_{n-1} \leq \tau_{n} \leq \tilde{\tau}_{n}$. The mean-squared truncation error is computed by

$$
\begin{aligned}
E\left\{\varepsilon_{N_{p}}^{2}\right\}= & E\left\{\int_{0^{-}}^{T_{\Omega}}\left|\Omega(\tau ; t)-\sum_{n=1}^{N_{p}} \alpha_{n}(t) \psi\left(\tau-\tau_{n}\right)\right|^{2} d \tau\right\} \\
= & \sum_{n=1}^{N_{p}} \int_{\tilde{\tau}_{n-1}}^{\tilde{\tau}_{n_{n}}} 2 Q\left(\tau^{\prime}\right) \\
& \int_{0^{-}}^{T_{\Omega}}\left[\psi\left(\tau-\tau^{\prime}\right)-\psi\left(\tau-\tau_{n}\right)\right]^{2} d \tau d \tau^{\prime} .
\end{aligned}
$$

In obtaining the numerical results we considered the specific case that $\tilde{\tau}_{n}$ 's were uniformly spaced and $\tau_{n}=\frac{1}{2}\left(\tilde{\tau}_{n-1}+\tilde{\tau}_{n}\right)$. Other details are as follows: rectangular symbol waveform with $\psi(\tau)=1$ for $\tau \in\left[0, T_{\psi}\right)$ and $\psi(\tau)=0$ otherwise was assumed; a truncated exponentially dispersive channel with $Q(\tau)=T_{\psi}^{-1} \exp \left(-\tau / T_{\psi}\right)$ for $\tau \in\left[0, T_{M}\right)$ and zero elsewhere, where $T_{M}=4 T_{\psi}$, was considered. The assumption of rectangular symbol waveform, although impractical, was used in this comparison because of simplicity; similar comparison results would be obtained for other choices of symbol waveform, such as Nyquist pulses.

Table I lists the numbers of terms of the truncated $\mathrm{K}-\mathrm{L}$ expansion $(N)$ required to achieve normalized mean-squared truncation errors of 10,5 , and $1 \%$ and those of the series expansion using the discrete-path approximation $\left(N_{p}\right)$. It is apparent that the value of $N_{p}$ is three to six times of the corresponding value of $N$, which indicates the computational advantage of using the truncated $\mathrm{K}-\mathrm{L}$ expansion over the discrete-path approximation in a simulation. It is also apparent 
TABLE I

Numbers of Terms Required to Achieve Given Normalized Mean-Squared Truncation Errors Using the Truncated K-L Expansion $(N)$ and Those Using the Series Expansion Based on the Discrete-PATH Approximation $\left(N_{p}\right)$

\begin{tabular}{ccc}
\hline $\begin{array}{c}\text { normalized mean-squared } \\
\text { truncation error }\end{array}$ & $N$ & $N_{p}$ \\
\hline $10 \%$ & 7 & 22 \\
$5 \%$ & 11 & 40 \\
$1 \%$ & 33 & 200 \\
\hline
\end{tabular}

that, as the required degree of simulation accuracy increases, the computational advantage of using the truncated $\mathrm{K}-\mathrm{L}$ expansion becomes more significant.

We did not include the Fechtel's series expansion [6] in the comparison because the series expansion of [6] is not mean-squared convergent to $\Omega(\tau ; t)$ for the communication system under consideration. There is a nonzero mean-squared error between $\Omega(\tau ; t)$ and its computed value obtained by the Fechtel's series expansion. The minimum mean-squared error is computed by (19) of [6]. We found that its normalized value is $33 \%$, so that it is not possible to represent the channel output by the Fechtel's series expansion with the accuracy requirements considered in this comparison.

\section{ApPlication to EfFicient Simulation}

We now apply the $\mathrm{K}-\mathrm{L}$ expansion of $\Omega(\tau ; t)$ to simulation of digital communications over a multipath Rayleigh fading WSSUS channel using an optimum receiver for signal processing.

\section{A. Communication System Description and Equivalent Discrete-Time Model}

The communication system under consideration is shown in Fig. 1. Let $\left\{I_{k}\right\}$ be the complex transmitted symbol sequence. The complex envelope of the transmitted signal, $s(t)$, is given by $s(t)=\sum_{m=-\infty}^{\infty} I_{m} \psi\left(t-m T_{s}\right)$ where $1 / T_{s}$ is the symbol transmission rate. The signal $s(t)$ is transmitted through the channel $h(\tau ; t)$. The complex envelope of the received signal, $r(t)$, is given by

$$
r(t)=n(t)+\sum_{m=-\infty}^{\infty} I_{m} \Omega\left(t-m T_{s} ; t\right)
$$

where $n(t)$ is the baseband-equivalent complex AWGN with the one-sided power spectral density $N_{0}$. An optimum receiver, which is a matched filter matched to $\Omega(\tau ; t)$ followed by sampling the matched filter output at a rate of $1 / T_{s}$ per second [9, ch. 6.3], is used to process $r(t)$. To implement an optimum receiver it is required that the channel is slowly varying relative to $2 T_{\Omega}$ so that the channel does not change appreciably during a time of $2 T_{\Omega}$, i.e., $\Omega(\tau ; t) \approx \Omega\left(\tau ; m T_{s}\right)$ where $\left|t-m T_{s}\right| \leq T_{\Omega}$. We assume that this requirement is satisfied. The complex-valued output of the optimum receiver at the $k$ th sampling instant, $\Xi_{k}$, is given by

$$
\Xi_{k}=\int_{k T_{s}}^{k T_{s}+T_{\Omega}} r(t) \Omega^{*}\left(t-k T_{s} ; k T_{s}\right) d t
$$

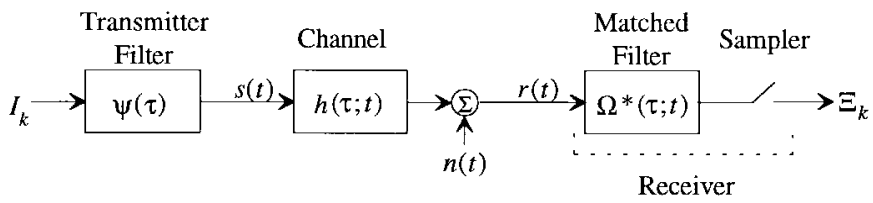

Fig. 1. The communication system model.

Since the discrete symbols are sent and the discrete-time sampled outputs of the receiver are also obtained at the same rate of $1 / T_{s}$ per second, one can construct an equivalent discrete-time (EDT) model that absorbs the combined effects of transmitter filtering, channel, receiver filtering and sampling into a single discrete-time input/output relationship. Details on the construction of the EDT model from the corresponding continuous-time communication system model are given by [9, ch. 4.7]. Here, only the results are stated. The EDT colorednoise filter model (Fig. 2) is realized by an FIR filter with time-variant tap gains. The output $\Xi_{k}$ can be expressed as

$$
\Xi_{k}=n_{k}+\sum_{m=-L}^{L} g_{m, k} I_{k-m}
$$

where $g_{m, k}$ 's are time-variant tap gains given by

$$
g_{m, k}=\int_{0^{-}}^{T_{\Omega}} \Omega^{*}\left(\tau ; k T_{s}\right) \Omega\left(\tau+m T_{s} ; k T_{s}\right) d \tau
$$

$\left\{n_{k}\right\}$ is the correlated complex Gaussian noise sequence with

$$
\begin{aligned}
& \frac{1}{2} E\left\{n_{u} n_{v}^{*}\right\} \\
& \quad= \begin{cases}N_{0} g_{v-u, k}, & |u-v| \leq L \text { and } k-L \leq u, v \leq k+L \\
0, & \text { otherwise }\end{cases}
\end{aligned}
$$

and $2 L+1$ is the number of tap gains. Since $g_{m, k}=0$ for $m \notin\{-L, \cdots, L\}$, we get

$$
L=\left\lceil\frac{T_{\Omega}}{T_{s}}\right\rceil-1 .
$$

For the sake of convenience, one usually whitens the noise sequence $\left\{n_{k}\right\}$ by filtering $\left\{\Xi_{k}\right\}$ with a noise whitening filter. Let $G_{k}(z)=\sum_{m=-L}^{L} g_{m, k} z^{-m}$ and $F_{k}(z)=$ $\sum_{m=0}^{L} f_{m, k} z^{-m}$ where $F_{k}(z)$ satisfies $F_{k}(z) F_{k}^{*}\left(z^{-1}\right)=$ $G_{k}(z)$ with roots of $F_{k}^{*}\left(z^{-1}\right)$ inside the unit circle. The output sequence of the noise whitening filter, $\left\{\Lambda_{k}\right\}$, is given by

$$
\Lambda_{k}=\eta_{k}+\sum_{m=0}^{L} f_{m, k} I_{k-m}
$$

where $\left\{\eta_{k}\right\}$ is a statistically independent, zero-mean complex Gaussian noise sequence with $\frac{1}{2} E\left\{\eta_{k} \eta_{k}^{*}\right\}=N_{0}$. The discretetime model that describes (23) is known as the EDT whitenoise filter model (Fig. 3).

The EDT model has been introduced because of the following reasons.

1) Straightforward implementation of simulation based on the continuous-time communication system model generally requires oversampling, which usually leads to considerable demand on computation. As pointed out 


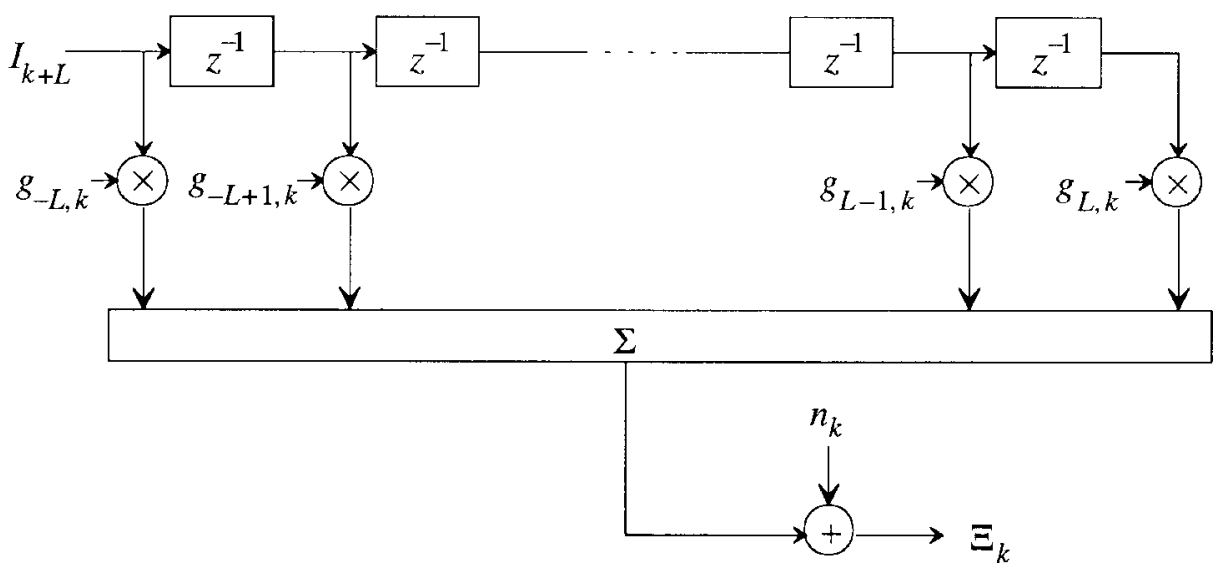

Fig. 2. The EDT colored-noise filter model.

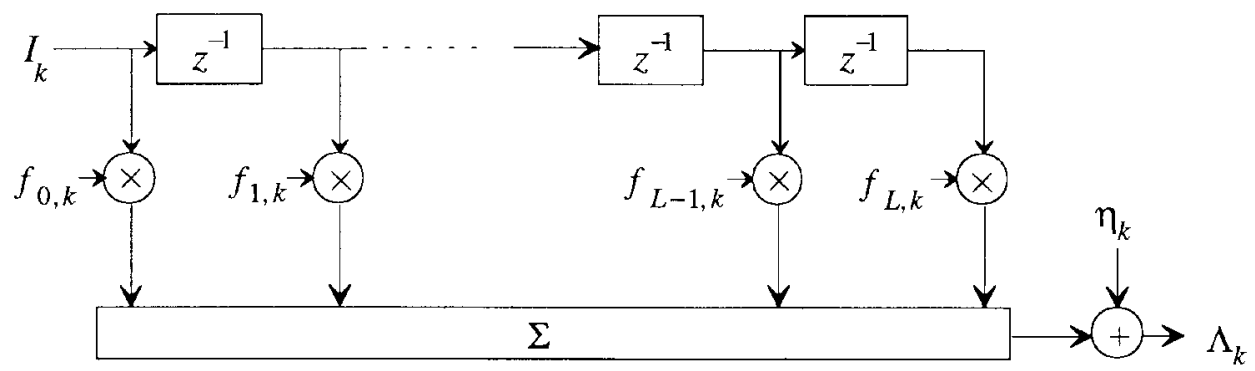

Fig. 3. The EDT white-noise filter model.

by Hoeher [10], the EDT model produces one output sample per input symbol at each sampling instant so that oversampling is eliminated in simulation. Hence, the EDT model is more efficient from the viewpoint of simulation.

2) The EDT white-noise filter model with deterministic tap gains is widely used in analytical works dealing with the presence of intersymbol interference (ISI), e.g., in the analysis of equalization algorithms [9, ch. 6.4] and in the study of using trellis coded modulation techniques for combating ISI [16]-[18]. By applying the results of these analytical works and by generating sample values of the tap gains using simulation techniques, one can obtain desired results for a fading dispersive channel.

\section{B. Implementation of the $K-L$ Expansion for Simulation}

It is desired to generate $g_{m, k}(20)$ based on the truncated $\mathrm{K}-\mathrm{L}$ expansion of $\Omega(\tau ; t)$, viz. $\Omega_{N}(\tau ; t)$ (8). Substituting (8) into (20) gives

$$
g_{m, k}=\mathbf{w}_{k}^{H} \boldsymbol{\Phi}_{m} \mathbf{w}_{k}
$$

where $(\cdot)^{H}$ is the complex conjugate transpose of the matrix, $\mathbf{w}_{k}$ is given by

$$
\mathbf{w}_{k}=\left[w_{1}\left(k T_{s}\right), w_{2}\left(k T_{s}\right), \cdots, w_{N}\left(k T_{s}\right)\right]^{T}
$$

and $\boldsymbol{\Phi}_{m}$ is an $N \times N$ matrix with the $(u, v)$ th element given by

$$
\Phi_{u, v}(m)=\int_{0^{-}}^{T_{\Omega}} \phi_{u}^{*}(\tau) \phi_{v}\left(\tau+m T_{s}\right) d \tau
$$

Note that $\Phi_{u, v}(-m)=\Phi_{v, u}^{*}(m)$ so that $g_{-m, k}=g_{m, k}^{*}$.
It is apparent that generating $\left\{g_{m}, k\right\}_{k}$ in simulation requires generating the complex Gaussian sequence $\left\{w_{n}\left(k T_{s}\right)\right\}_{k}$. The generation of the latter sequence can be accomplished by a number of methods such as filtering and linear transformation methods [19, ch. 3], the Fourier series expansion technique [7] and the Monte Carlo (MC) technique described in [11]. After $\left\{w_{n}\left(k T_{s}\right)\right\}_{k}, n=1,2, \cdots, N$, are generated, $\left\{g_{m, k}\right\}_{k}$, $m=0,1, \cdots, L$ can be computed by (24), and $\left\{g_{-m, k}\right\}_{k}$. can be easily obtained since $g_{-m, k}=g_{m, k}^{*}$. The $\left\{f_{m, k}\right\}_{k}$, $m=0,1, \cdots, L$ can be obtained from $\left\{g_{m}, k\right\}_{k}$ with some algebraic manipulations. After the white noise sequence $\left\{\eta_{k}\right\}$ is generated by a standard technique, $\left\{\Lambda_{k}\right\}$ can be computed for a given $\left\{I_{k}\right\}$ by using (23).

\section{Comparison with Other Simulation Techniques}

Simulation runs were performed to compare the computation times required by: i) the proposed simulation technique which employs the $\mathrm{K}-\mathrm{L}$ expansion, ii) the one that approximates the channel by a number of discrete multipaths with uniformly spaced $\tilde{\tau}_{n}$ 's, and iii) the Hoeher's simulation technique [10]. A 5\% normalized mean-squared error in the generation of $\Omega(\tau ; t)$ was assumed in order to ensure that accuracy in the generation of $g_{m, k}$ by the three techniques under consideration was (almost) the same. The communication system we employed in the simulation was the same as the one we considered in Section IV. In addition, we assumed that i) $T_{\psi}=T_{s}$ (so that $L=4$ ), and ii) $S(f)=$ $\left[\pi f_{D_{\max }} \sqrt{1-\left(f / f_{D_{\max }}\right)^{2}}\right]^{-1}$ where the maximum Doppler frequency $f_{D_{\max }}$ was given by $f_{D_{\max }}^{-1}=100 T_{s}$. Five tap gains $\left(g_{m, k}, m=0,1, \cdots, 4\right)$ were generated in a run, and a total 
TABLE II

Set-Up Times And Run Times Required By the Three Simulation TechniQues

\begin{tabular}{ccc}
\hline & Set-up time & $\begin{array}{c}\text { Run time } \\
\text { for } 10^{4} \\
\text { simulation runs }\end{array}$ \\
\hline K-L expansion & $12.6 \mathrm{~s}$ & $76 \mathrm{~s}$ \\
Discrete-path approx. & $0.1 \mathrm{~s}$ & $272 \mathrm{~s}$ \\
Hoeher's technique & $5.8 \mathrm{~s}$ & $16944 \mathrm{~s}$ \\
\hline
\end{tabular}

of $10^{4}$ runs $\left(k=1,2, \cdots, 10^{4}\right)$ were performed for each technique. A SUN SPARC 5 was used, and simulation was done using MATLAB.

Procedures for generating $g_{m, k}$ values using the three considered techniques are described in Appendix II. It is shown that for each of these techniques the $g_{m, k}$ value can be computed by an appropriate quadratic form so that the number of computation roughly depends on $N_{m}^{2}$ where $N_{m} \times N_{m}$ is the dimension of the matrix in the quadratic form. It is also shown that values of $N_{m}$ are 11, 40, and 410 for the technique using the $\mathrm{K}-\mathrm{L}$ expansion, the one using the discretepath approximation, and the Hoeher's technique, respectively. The result that $N_{m}$ of the first technique is smallest indicates the computational efficiency of using the $\mathrm{K}-\mathrm{L}$ expansion over the other two techniques in simulation. We also remark that the large difference of $N_{m}$ values between the $\mathrm{K}-\mathrm{L}$ expansion approach and Hoeher's technique results in a large difference between their computation requirements, as demonstrated by the numerical results that follow.

Table II lists the set-up times (times required to compute eigenvalues, eigenfunctions, fill up matrices, etc.) and run times (times taken to generate $g_{m, k}$ values in $10^{4}$ simulation runs) for the three techniques under consideration. Note that the set-up procedure is done once only so that the set-up time is a fixed value independent of the number of simulation runs. As expected, the $\mathrm{K}-\mathrm{L}$ expansion approach required the longest set-up time due to the considerable computation involved in the evaluation of eigenvalues and eigenfunctions. However, this greater computational requirement was offset by the significant reduction of the run time required to perform $10^{4}$ simulation runs. The total computation times (set-up time + run time) were 89,272 , and 16950 s for the $\mathrm{K}-\mathrm{L}$ expansion approach, the discrete-path approximation approach, and the Hoeher's technique, respectively. The numerical results confirm the expected simulation efficiency of the $\mathrm{K}-\mathrm{L}$ expansion approach over the other two techniques.

\section{CONCLUSIONS}

The K-L expansion of the WSSUS channel output has been derived. Numerical results have shown that under the same mean-squared truncation error the number of terms required by the $\mathrm{K}-\mathrm{L}$ expansion is less than that required by the discretepath approximation of the channel in computing the channel output. It indicates the computational efficiency of using the $\mathrm{K}-\mathrm{L}$ expansion in simulation. It has also been found that the computational advantage of using the $\mathrm{K}-\mathrm{L}$ expansion is more significant if a higher degree of simulation accuracy is required.
The derived expansion has been applied to develop an efficient simulation technique for digital communications over a multipath Rayleigh fading WSSUS channel using an optimum receiver. An expression for computing the time-variant tap gain of the EDT model of the communication system has been derived. The simulation efficiency of the proposed technique over the technique based on approximating the channel by a number of discrete multipaths and the Hoeher's simulation technique has been demonstrated.

\section{APPENDIX I}

\section{NUMERICAL SOLUTION OF (9)}

The following method is adapted from [3, Appendix] and [4, ch. 2]. Consider the integral eigenvalue problem

$$
\int_{0^{-}}^{T_{\Omega}} R(\tau, \xi) \phi(\xi) d \xi=\lambda \phi(\tau) .
$$

The $\left\{\lambda_{n}, \phi_{n}(\tau)\right\}, n=1,2, \cdots, N$, are any $N$ sets of solutions to this problem. It is assumed that $R(\tau, \xi), \tau, \xi \in$ $\left[0, T_{\Omega}\right)$, is continuous. Using Simpson's rule of numerical integration with $N_{I}\left(N_{I} \geq N\right)$ points where $N_{I}$ is an odd integer, one can express this integral eigenvalue problem as an algebraic eigenvalue problem

$$
\operatorname{RD} \phi=\lambda \phi .
$$

In the expression, $\mathbf{D}$ and $\mathbf{R}$ are $N_{I} \times N_{I}$ matrices with $(k, \ell)$ th elements given by

$$
[\mathrm{D}]_{k, \ell}= \begin{cases}\frac{\Delta}{3}, & k=\ell=1 \text { or } k=\ell=N_{I} \\ \frac{4 \Delta}{3}, & k=\ell=2,4,6, \cdots, N_{I}-1 \\ \frac{2 \Delta}{3}, & k=\ell=3,5,7, \cdots, N_{I}-2 \\ 0, & k \neq \ell\end{cases}
$$

respectively, and $\phi$ is an $N_{I} \times 1$ eigenvector with $[\phi]_{k}=\phi\left(\tau_{k}\right)$ where $\Delta=T_{\Omega} /\left(N_{I}-1\right), \tau_{k}=(k-1) \Delta$ and $\xi_{\ell}=(\ell-1) \Delta$. This algebraic eigenvalue problem can be solved by standard techniques.

\section{APPENDIX II}

\section{GENERATION OF $g_{m, k}$ BY THE TECHNIQUES CONSIDERED IN SECTION V-C}

In the $\mathrm{K}-\mathrm{L}$ expansion approach, the method described in Appendix I with $N_{I}=101$ was used to compute the eigenvalues and eigenfunctions. The $g_{m, k}$ value was computed by (24) with $N=11$ (Table I). Let $R_{\psi \psi}(\xi)=$ $\int_{0^{-}}^{T_{\psi}} \psi(\tau) \psi(\tau+\xi) d \tau$. In the discrete-path approximation approach, $g_{m, k}$ was computed by $g_{m, k}=\boldsymbol{\alpha}_{k}^{H} \mathbf{Y}_{m} \boldsymbol{\alpha}_{k}$ where $\boldsymbol{\alpha}_{k}=\left[\alpha_{1}\left(k T_{s}\right), \alpha_{2}\left(k T_{s}\right), \cdots, \alpha_{N_{p}}\left(k T_{s}\right)\right]^{T}$ and $\mathbf{Y}_{m}$ is an $N_{p} \times N_{p}$ matrix with $\left[\mathbf{Y}_{m}\right]_{u, v}=R_{\psi \psi}\left(\tau_{u}-\tau_{v}+m T_{s}\right)$. In the computation, $N_{p}=40$ was used (Table I). The $\mathbf{w}_{k}$ and $\boldsymbol{\alpha}_{k}$ were generated by the MC method described as follows. For a complex zero-mean Gaussian random process 
$c(t)$ that satisfies $E\left\{c(t) c^{*}(t+\Delta t)\right\}=D(\Delta t)$, it is known that [11] $c(t)=\lim _{N_{\mathrm{RAY}} \rightarrow \infty} c_{N_{\mathrm{RAY}}}(t)$ where $c_{N_{\mathrm{RAY}}}(t)=$ $N_{\mathrm{RAY}}^{-1 / 2} \sum_{n=1}^{N_{\mathrm{RAY}}} \exp j\left(\theta_{n}-2 \pi \mu_{n} t\right)$. In the expression, $\theta_{n}$ 's are i.i.d. random variables uniformly distributed over $[0,2 \pi)$, and $\mu_{n}$ 's are i.i.d. random variables with $p\left(\mu_{n}\right)=$ $S\left(\mu_{n}\right) / \int_{-\infty}^{\infty} S(\mu) d \mu$. In the MC method, $c(t)$ is generated by $c_{N_{\mathrm{RAY}}}(t)$ with randomly generated seeds $\theta_{n}$ 's and $\mu_{n}$ 's. The value of $N_{\mathrm{RAY}}$ determines the accuracy in approximating a complex Gaussian distribution. We used $N_{\mathrm{RAY}}=10$ in the simulation. In the Hoeher's method, $g_{m, k}$ was computed by $g_{m, k}=\boldsymbol{\beta}_{k}^{H} \mathbf{Z}_{m} \boldsymbol{\beta}_{k}$ where $\boldsymbol{\beta}_{k}$ is an $N_{H} \times 1$ vector with $\left[\boldsymbol{\beta}_{k}\right]_{n}=N_{H}^{-1 / 2} \exp j\left(\theta_{n}+2 \pi \mu_{n} k T_{s}\right)$ and $\mathbf{Z}_{m}$ is an $N_{H} \times N_{H}$ matrix with $\left[\mathbf{Z}_{m}\right]_{u, v}=R_{\psi \psi}\left(\gamma_{u}-\gamma_{v}+m T_{s}\right)$. In the above, $N_{H}$ is an integer, $\theta_{n}$ and $\mu_{n}$ follow the same definitions in defining $c_{N_{\mathrm{RAY}}}(t)$, and $\gamma_{n}, n=1,2, \cdots, N_{H}$, are i.i.d. random variables with $p\left(\gamma_{n}\right)=Q\left(\gamma_{n}\right) / \int_{-\infty}^{\infty} Q(\gamma) d \gamma$. Since an expression for computing the mean-squared error in terms of $N_{H}$ was not provided by Hoeher [10], and to the authors' best knowledge [11] is the only literature that enables one to compute this parameter, we apply the result of [11] in its computation. It has been shown [11] that Hoeher's method may be regarded as a technique that generates $g_{m, k}$ by the discrete-path approximation of the channel with $\tilde{\tau}_{n}$ 's selected such that values of $\int_{\tilde{\tau}_{n-1}}^{\tilde{\tau}_{n}} Q(\tau) d \tau$ are equal $\forall n=1,2, \cdots, N_{p}$, and by generating the complex path gain using the MC method. Therefore, (16) can be used to compute $E\left\{\varepsilon_{N_{p}}^{2}\right\}$ for the Hoeher's method. Assuming $\tau_{n}=\frac{1}{2}\left(\tilde{\tau}_{n-1}+\tilde{\tau}_{n}\right)$, we found that $N_{p}=41$. Since $N_{H}=N_{\mathrm{RAY}} N_{p}$ [11], we used $N_{H}=410$ in the simulation.

\section{ACKNOWLEDGMENT}

The authors would like to thank one of the anonymous reviewers for indicating that it is also possible to carry out efficient simulation in the frequency domain using the $\mathrm{K}-\mathrm{L}$ expansion derived in [3] and [4].

\section{REFERENCES}

[1] H. P. Kramer, "On best approximations of random processes, et al.," IRE Trans. Inform. Theory, vol. IT-6, pp. 52-53, Mar. 1960.

[2] J. B. Thomas, An Introduction to Communication Theory and Systems. New York: Springer-Verlag, 1988.

[3] M. V. Clark, L. J. Greenstein, W. K. Kennedy, and M. Shafi, "Matched filter performance bounds for diversity combining receivers in digital mobile radio," IEEE Trans. Veh. Technol., vol. 41, pp. 356-362, Nov. 1992.

[4] M. V. Clark, "Diversity and equalization in digital cellular radio," Ph.D. thesis, University of Canterbury, New Zealand, May 1995.

[5] M. V. Clark, L. J. Greenstein, W. K. Kennedy, and M. Shafi, "Optimum linear diversity receivers for mobile communications," IEEE Trans. Veh. Tech., vol. VT-43, pp. 47-56, Feb. 1994

[6] S. A. Fechtel, "A novel approach to modeling and efficient simulation of frequency-selective fading radio channels," IEEE J. Select. Areas Comm., vol. 11, pp. 422-431, Apr. 1993.

[7] P. M. Crespo and J. Jiménez, "Computer simulation of radio channels using a harmonic decomposition technique," IEEE Trans. Veh. Tech., vol. 44, pp. 414-419, Aug. 1995.

[8] M. Visintin, "Karhunen-Loève expansion of a fast Rayleigh fading process," Elec. Lett., vol. 32, pp. 1712-1713, Aug. 1996.
[9] J. G. Proakis, Digital Communications, 2nd ed. New York: McGrawHill, 1989.

[10] P. Hoeher, "A statistical discrete-time model for the WSSUS multipath channel," IEEE Trans. Veh. Technol., vol. 41, pp. 461-468, Nov. 1992.

[11] K. W. Yip and T. S. Ng, "Efficient simulation of digital transmission over WSSUS channels," IEEE Trans. Comm., vol. 43, pp. 2907-2913, Dec. 1995.

[12] P. A. Bello, "Characterization of randomly time-variant linear channels," IEEE Trans. Commun. Sys., vol. 11, pp. 360-393, Dec. 1963.

[13] W. B. Davenport, Jr. and W. L. Root, An Introduction to the Theory of Random Signals and Noise. New York: McGraw-Hill, 1958 (also reprinted by the IEEE Press, New York, 1987).

[14] H. Cohen, Mathematics for Scientists and Engineers. Englewood Cliffs, NJ: Prentice Hall, 1992.

[15] C. T. H. Baker, The Numerical Treatment of Integral Equations. Oxford, England: Oxford Univ. Press, 1977.

[16] P. R. Chevillat and E. Eleftheriou, "Decoding of trellis-encoded signals in the presence of intersymbol interference and noise," IEEE Trans. Comm., vol. 37, pp. 669-676, July 1989.

[17] W. H. Sheen and G. L. Stüber, "Error probability of reduced-state sequence estimation for trellis-coded modulation on intersymbol interference channels," IEEE Trans. Commun., vol. 41, pp. 1265-1269, Sept. 1993

[18] S. J. Simmons, "Alternative trellis decoding for coded QAM in the presence of ISI," IEEE Trans. Commun., vol. 42, pp. 1455-1459, Feb./Mar./Apr. 1994.

[19] M. C. Jeruchim, P. Balaban, and K. S. Shanmugan, Simulation of Communication Systems. New York: Plenum, 1992.

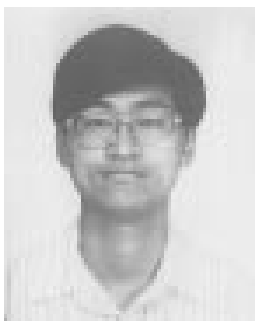

Kun-Wah Yip (M'96) received the B.Eng. (Hons) and $\mathrm{Ph} . \mathrm{D}$. degrees in electrical engineering from The University of Bradford, England, in 1991 and the University of Hong Kong in 1995, respectively.

In 1995 he spent half a year as a Research Associate at The University of Hong Kong. His main duty was the design and simulation of a directsequence spread-spectrum communication system using a novel multiple-access technique. Since 1996 he has been with the University of Hong Kong as a Postdoctoral Fellow. His research interest is on spread-spectrum communications, channel and system modeling for the purpose of efficient simulation, and theory and techniques for communications over frequency-selective fading channels.

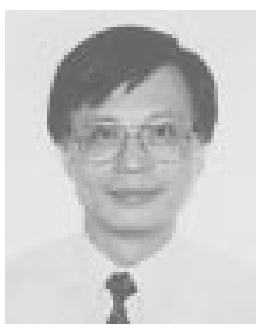

Tung-Sang Ng received the B.Sc.(Eng.) degree from the University of Hong Kong in 1972, and the M.Eng.Sc. and Ph.D. degrees from the University of Newcastle, Australia, in 1974 and 1977, respectively, all in electrical engineering.

He was employed as an Electrical Engineer by BHP Newcastle Iron and Steel Works, Australia, from 1972 to 1974 . From 1977 to 1990 he was a Lecturer, then Senior Lecturer and Reader in the Department of Electrical and Computer Engineering, The University of Wollongong, Australia. Currently, he is holding the chair of Electronic Engineering at the University of Hong Kong. His current research interests include spread-spectrum techniques, digital signal processing, mobile communication systems, and engineering applications of artificial intelligence. He has published over 130 journal and conference papers.

Dr. $\mathrm{Ng}$ is a Fellow of IEE, HKIE, and IEAust. He is a Co-Chairman of the 1996 2nd IEE International Conference on Personal, Mobile and Spread Spectrum Communications, and a General Co-Chairman of the 1997 IEEE International Symposium on Circuits and Systems to be held in Hong Kong. He is also a Regional Editor for the international journal, Engineering Applications of Artificial Intelligence (Pergamon Press). 